\title{
LA PERTINENCIA DE LOS MÉTODOS DE ENSEÑANZA-APRENDIZAJE DESDE LA TELEOLOGÍA DE LA EDUCACIÓN
} Relevance of learning and teaching methods from the teleology of education

\author{
JONATHAN A. VIVAS $H .{ }^{*}$ \\ jvivas_pegasus@hotmail.com \\ Universidad Politécnica Salesiana del Ecuador / Quito-Ecuador
}

\begin{abstract}
Resumen
Los métodos de enseñanza-aprendizaje se hallan relacionados con la finalidad de la educación. El método contribuye con la educación para alcanzar un principio básico de humanizar al hombre. Desde ahí, se entiende que el conocer sigue un proceso para la apropiación del conocimiento que exige del educando adoptar reglas para seguir aprendiendo, es por eso que el educando debe comprender por qué aprende y qué proceso ha de seguir para alcanzar el conocimiento. De igual manera es importante mencionar que la relación enseñanza-aprendizaje es dialéctica, se inscribe en una diaologicidad, donde cada uno, tanto quien enseña como quien aprende, hace uso de métodos para promover nuevos saberes. Luego, desde la competencia educativa se evidencia el acceso a nuevos aprendizajes, por las habilidades y destrezas adquiridas para el cumplimiento del telos educativo, la humanización del hombre, porque es visto como persona que se educa y se relaciona con otros; es capaz de transformar su entorno.
\end{abstract}

Palabras claves

Pertinencia, competencia, educación, teleología, métodos, enseñanza, aprendizaje.

Abstract

Learning and teaching methods are related to the goals of education. Methods help education to fulfill its basic principle: the humanization of man. From this follows that knowledge is a process of appropriation that requires from the student the adoption of rules to make of learning a continuum; understanding why they learn and what kind of process needs to be followed to reach knowledge. Learning - teaching relationship is a dialectic one: who learns and who teaches must use methods to promote new knowledge. Thus, educational competency evidences the access to new learnings and the acquisition of new skills to fulfill the finality of education: the humanization of man insofar is a person that can be educated and a person that establishes relationships with others, capable of transform their environment.

Keywords

Relevance, competition, education, teleology, methods, teaching, learning.

Forma sugerida de citar: Vivas H., Jonathan A. (2015). La pertinencia de los métodos de enseñanza-aprendizaje desde la teleología de la educación. Sophia: colección de Filosofía de la Educación, 19 (2), pp. 73-92.

* Licenciado en Filosofía y Pedagogía por la Universidad Politécnica Salesiana del Ecuador. Docente en el Colegio Virtual Iberoamericano. 


\section{Introducción}

Las reflexiones en torno a 'la pertinencia de los métodos de enseñanzaaprendizaje desde la teleología de la educación' hacen posible abrirse campo en la educación para corroborar hipótesis sobre lo que se considera una prioridad para los docentes: los métodos son un medio para alcanzar la finalidad de la educación. La humanización del hombre. Es necesario detenerse y pensar por separado tanto los métodos de quien enseña como de quien aprende.

Este tema tiene como objetivo evidenciar la adquisición de destrezas y habilidades a partir de métodos de enseñanza-aprendizaje, en términos de competencia, tanto para quien enseña como para quien aprende. Es comprensible que en esta relación ambos contribuyan al desenvolvimiento de la actividad del otro. Así mismo se critica la utilidad de ciertos métodos a partir de la diversidad porque no todos aprenden de igual manera.

Estas ideas se desarrollan con base en la solución del problema que se refiere al uso repetitivo de métodos en la enseñanza-aprendizaje que impiden la correcta adquisición de destrezas y habilidades para seguir aprendiendo; para solucionar esto, se sugiere que a partir de la relación entre el educador y el educando se prevea la mejor utilización de los métodos para cumplir con la finalidad de la educación.

El presente trabajo es importante por la presencialidad del acto educativo donde surgen diversos cuestionamientos, uno de ellos se instaura en la diversidad de aprendizajes, es decir, que no todos los estudiantes aprenden de igual manera por lo que no se puede hacer uso de un mismo método todo el tiempo, habría que variar dependiendo de muchos factores que inciden en el proceso educacional. Además, se hace hincapié en la actualización docente, ya que la sociedad impone nuevos retos para el docente.

En la actualidad es notable que los docentes desarrollen métodos para cumplir con los objetivos propuestos, pero no con la finalidad de cumplir con el plan de clase, sino más bien con el propósito de que los estudiantes aprendan. De igual manera los estudiantes van acogiendo estos métodos, así como los conocimientos, de modo que, en ausencia del docente, pueden seguir aprendiendo. Si la educación tiene como fin último la humanización del hombre (Fullat, 2002), en consecuencia la relación entre el docente-discente deberá estar enfocada en este telos.

Se establece una breve conjetura sobre la pertinencia de los métodos de enseñanza-aprendizaje donde 'los docentes contribuyen a mejorar la calidad de la educación al ofrecer reglas (métodos) para estructuración fuerte del pensamiento, todo esto mediante el correcto uso de los métodos para viabilizar eficazmente el aprendizaje considerando las distintas formas de aprender de los estudiantes. 
Es interesante mostrar los métodos a partir de la relación entre la enseñanza-aprendizaje porque ofrecen aportes de mucha ayuda para el campo educativo, puesto que permiten la criticidad sobre lo que se está haciendo en el acto educativo. Para lograr este cometido se hace uso de una metodología descriptiva y crítica, resultado de una investigación bibliográfica fundamentada.

Esta temática se encuentra distribuida así: Primero, se hace hincapié en la apropiación del conocimiento como un proceso cognitivo en donde se van adquiriendo reglas, a raíz de los métodos de enseñanza, de manera que un estudiante pueda aprender eficazmente porque posee una estructura cognitiva fuerte y esto le permite el acceso a conocimientos complejos; en segundo lugar, se muestra la relación enseñanza-aprendizaje, pero vista desde la utilización de métodos donde los docentes y los estudiantes usan metodologías para cumplir con sus objetivos, ambos enseñan y aprenden en el acto educativo, según Álvarez et al. (2008); como tercer punto se encuentra la competencia educativa a partir de los métodos de enseñanza-aprendizaje, lo cual significa que los entes que intervienen en el acto educativo se vuelven competentes en la medida en que se desempeñan, no solo en el aula de clase, sino también en su entorno, se alude a la educación para 'enfrentar las incertidumbres' (Morin, 1999); por último, aparece la teleología de la educación como resultado de un proceso de enseñanza-aprendizaje pertinente, donde los valores juegan un rol determinante en el que se está educando a la persona y los medios que se utilizan para que se humanice y se relacione con los otros (Saavedra, 2007).

\section{Métodos de enseñanza-aprendizaje en el proceso de apropiación del conocimiento}

La apropiación del conocimiento conlleva un proceso que no se agota en la institucionalización de los saberes. Los conocimientos que se van adquiriendo a lo largo de la vida son útiles porque cada ser humano es consciente de su proceso de aprendizaje y más aún, se entiende que este proceso es personal y no colectivo, puesto que se dice de "la relación mente-cerebro, que la mente se lanza y entra en el mecanismo de la física, eso es el carácter incorporado y desde allí se hace posible comprender su mecanismo o funcionamiento" (Sanmartin, 2014: 122). La afirmación de este primer argumento se asemeja a la acción del ser humano para conocer el mundo a través de reglas y métodos en la consecución de algo: el conocimiento.

El individuo empieza de cero (en cuanto aprendizajes) y va adquiriendo un cúmulo de reglas para estructurar su conocimiento a nivel cere- 
bral, por eso es que "la institución educativa es el ámbito de desarrollo de los procesos de enseñanza y de aprendizaje" (Álvarez et al., 2008), debido a que, en primera instancia, los métodos que son útiles en el pre-kinder se vuelven complejos por el acceso a niveles más altos de aprendizaje, es decir, en la medida en que la estructura del pensamiento se complejiza. Jerome Bruner, en la 'Revolución Cognitiva', especificaba que debía darse un "currículo en espiral, la idea de este currículo en espiral lleva a pensar en una especie de estructuración" (Guilar, 2009: 238). Se establece una estructura donde el planteamiento acepta que un estudiante debería trabajar los mismos contenidos, ideas o conceptos, cada vez con mayor profundidad.

En este proceso hay que ser cuidadosos porque puede darse un empobrecimiento a causa del parcelamiento referencial y es que los métodos utilizados en la enseñanza, muchas veces, no consideran el contexto social:

Lo que se presenta de la realidad aparece en forma puntual, aislado de otros elementos, como algo en sí mismo, válido hasta sus límites. Se procede a una permanente descontextualización social, como si los seres y los acontecimientos fueran autosuficientes, y nadie tuviera que ver con nadie. A mayor descontextualización, a mayor parcelamiento, menos conocimiento de las causas, de las reales conexiones sociales que caracterizan a una formación social (Prieto Castillo, 1985: 122).

Esta relación de enseñanza-aprendizaje está sujeta a la observancia del contexto y desde ahí se hace más fácil aplicar métodos que aseguran la adquisición de conocimientos, más no como una tabula rasa ${ }^{1}$ sino más bien como actividad; el niño es un sujeto activo que aprende y se apropia de su entorno. Así mismo, va apropiándose de ciertas reglas para seguir aprendiendo, entendiendo su contexto y a sí mismo en él.

Supóngase que un individuo se encuentra aislado (sin la posibilidad para acceder a la escuela) y se introduce al mundo para conocer, pero tiene un total desconocimiento de reglas o métodos de aprendizaje, sin embargo, aquel niño va a aprender, pero no va a seguir un proceso símil al escolarizado, por lo que tendrá complicaciones en hacer relaciones entre las cosas para afianzar su conocimiento en relación a su entorno, de ahí la comprensión de que "todo conocimiento nuevo supone una abstracción, porque, a pesar de la reorganización que entraña, nunca constituye un comienzo absoluto, sino que extrae sus elementos de alguna realidad anterior" (Piaget, 1980: 138). Las reglas son muy útiles, pero no lo son todo, además la alternancia de los métodos con los que se trabaja en la escuela, por parte de quien enseña, son válidos en la medida en que permiten al individuo hacer uso de ellas para ser consciente de su aprendizaje y el beneficio que puede sacar del mismo para transformar su realidad. 
Cuando se habla de apropiación del conocimiento inmediatamente se pasa al campo de referencialidad de saberes, pues a través del lenguaje se hace posible dicha empresa, donde "el lenguaje supone por tanto, una comunidad de interpretación, intérpretes actuales e intérpretes del pasado que contribuyeron en la configuración de este tesoro de conocimientos" (Rolón et al., 1997: 51), siendo de mayor interés la manera en la que el individuo hace suyos determinados conocimientos. Si bien es cierto, cuando se conoce, se empieza a hacer relaciones, pero se sigue un camino o pautas para pasar la realidad al lenguaje, del pensamiento e ideas.

Para ahondar más en el asunto se presenta al individuo frente al objeto en donde hay una relación sujeto-objeto y el centro es el sujeto quien conoce -a diferencia del objetocentrismo- apareciendo en primer lugar; para conocer se hace uso de métodos que pueden ayudar al sujeto en su investigación, organización y transmisión de conocimientos. Así, estos métodos no son únicos y tampoco se encuentran predeterminados por un grupo específico, antes bien, varían dependiendo de los estímulos que pueda recibir el individuo, ya sea de forma auditiva, visual o kinestésica. Se insiste en que la realidad se traslada al lenguaje para una mejor comprensión del mundo, confirmando que:

El lenguaje supone un concepto de realidad objetiva, una realidad que podría verse, tocarse y señalarse, operar con ella y en ella ostensivamente. Aunque lo que permite llamarla realidad y decir que se puede operar con ella es la interpretación que construimos de ella en comunidad, de nosotros mismos y del conocimiento. Esta interpretación que sólo se puede comunicar en un lenguaje (presentativo, intencional, designativo) $[\ldots]$, se basa en la posesión de un concepto mundo que compartimos y conocemos (Rolón, et al., 1997: 51-52).

La comprensión del mundo es vista como un proyecto que se lleva a cabo mediante una serie de métodos claramente identificables que hacen posible la actualización de su contenido. Consecuentemente el accionar del individuo se da por la interpretación, donde, por un lado está lo presentativo que se entiende como lo que acontece, lo que se muestra y, mediante la observación, es fácil constatar este hecho, además de hacer uso de otros medios receptores propios de los sentidos; luego se presenta lo intencional, pues cada sujeto se sumerge en el mundo porque así lo quiere, tiene la voluntad para entenderlo y sigue un camino (uso de métodos de aprendizaje) para la objetivación de la realidad; por último, queda lo designativo, que se traduce en la referencialidad entendiendo, desde allí, que "la ciencia o los enunciados de la ciencia no son vacuos [...]. Por la vía de la referencialidad se llega a la esencia de las cosas, de sustancias con sus propiedades" (Sanmartín, 2014: 125), entendiendo que el individuo para conocer necesita de 
la realidad, de las cosas, para comprenderlas y apropiarse de ellas como realidades abstractas; sólo el hombre es capaz de valorar, por su conocimiento, la realidad que lo rodea, ya sea para mejorar o destruir.

En párrafos anteriores, se hacía mención a los procesos metodológicos que ayudarán al individuo en su propósito de conocer y de igual manera la voluntad que debe tener. Luego, para que haya esa predisposición, es necesario considerar determinadas variables a nivel de la estructura cognitiva como:

1) La disponibilidad en la estructura cognitiva del estudiante de unas ideas de anclaje específicamente pertinentes con un nivel óptimo de inclusividad, generalidad y abstracción; 2) la medida en que estas ideas se pueden discriminar de conceptos y principios tanto similares como diferentes (pero potencialmente confundibles) del material de aprendizaje; $y$ 3) la estabilidad y la claridad de las ideas de anclaje (Ausubel, 2002: 40).

Ausubel presenta variables que se traducen en la forma de conocer del individuo. Ahora, supóngase que un estudiante adquiere métodos, entre ellos hace uso del método heurístico y el método de resolución de problemas, para ambos casos hay ideas de anclaje específicamente pertinentes con las cuales va a hilar lo previo con lo nuevo y, desde allí se puede hablar de la abstracción que hace de la realidad; es cierto que sabe discriminar, es decir, que puede considerar unas ideas más importantes que otras, puesto que al tomar elementos de la realidad ya empezó a hacer esta discriminación a nivel cognitivo; por último, está la claridad y estabilidad de las ideas, de manera que haya un equilibro entre los conocimientos previos y los que acaba de adquirir. Los métodos siguen siendo fundamentales porque se traducen en reglas para poder aprender.

El aprendizaje, a nivel de la estructura cognitiva, exige adoptar métodos que son importantes para aprender, para apropiarse del conocimiento. No puede haber una limitación a la hora de escoger uno o más métodos para conocer, ya que depende en gran medida de los estilos de aprendizaje que cada individuo posea, así mismo haciendo uso de técnicas que permitan facilitar la adquisición de esos conocimientos de forma más sencilla; por ejemplo: un estudiante puede hacer uso de un método de exposición, de acuerdo a su estilo de aprendizaje visual, aunado a la técnica del organizador gráfico y logrará entender conceptos específicos de cualquier materia. Los 'mapas mentales' son considerados como "organizadores que se presentan con un nivel de abstracción, generalidad e inclusividad más elevado que el material nuevo que se debe aprender" (Ausubel, 2002: 41), de tal modo que el aprendizaje de nuevos conocimientos es mucho más efectiva cuando se sigue un determinado con el cual se está familiarizado. 
Es importante tener presente que los métodos son utilizados tanto para la enseñanza como para el aprendizaje y, en muchos casos, son herramientas útiles de acuerdo a los estilos de aprendizaje, puesto que no puede afirmarse que todos aprenden de la misma manera; desde allí se hace visible la necesidad de llevar a cabo procesos metodológicos, tanto para aprender como para enseñar. 'No todos los cerebros se estructuran de la misma forma, incluso compartiendo la misma clase y aprendiendo el mismo currículo o, más aún, con las mismas experiencias compartidas del contexto'2.

Los métodos, al interior del acto educativo, deben ser considerados como un medio y no el fin, por lo tanto, son importantes en el proceso de aprendizaje y a continuación se defiende la validez que presentan los métodos para los estudiantes y para los docentes. El docente sigue aprendiendo porque necesita actualizar sus conocimientos para hacer frente a la tarea de enseñar y el estudiante aprende porque requiere darle sentido a su existencia para poder cumplir con la compleja tarea de humanizarse.

\section{Los métodos desde la relación enseñanza-aprendizaje}

Los docentes son una pieza fundamental en la educación y, por ello, esta actividad u oficio debería ser considerada muy importante para todas las épocas. No parecería oportuno negar su valor en el constructivismo, ya que, aun como guía (Bruner, 1963) sigue teniendo un rol importantísimo, más no porque sea poseedor de los diferentes conocimientos, sino por el hecho de que puede aportar mucho al aprendizaje del individuo $y$, éste último por tener conocimientos previos, puede sorprender al docente. El rol docente solo necesita actualización y es su deber mantenerse así todo el tiempo, justamente para alcanzar una educación de calidad.

Al pensar en el rol docente, en la responsabilidad que tiene sobre sí para lograr que sus discentes aprendan, se evidencia que los estudiantes hacen uso de métodos que aprenden de quien les enseña, y en la medida en que hacen uso de estos métodos, así mismo, el docente tiene que hacer posible que los estudiantes aprendan de forma consciente. Los discentes harán uso de ellos para aprender en ausencia del maestro, para afrontar el mundo y entenderlo. Es necesaria una aclaración, se refiere a la pedagogía del docente, la cual "permite situarse en cada época para responder a las necesidades o situaciones que se presentan" (Fullat, 2002: 9), desde allí ya se va prefigurando el uso de métodos de acuerdo a un contexto. Sépase lo que sucede a nivel cognitivo.

La docencia es entendida, en estas reflexiones a partir de un antiguo proverbio chino el cual decía: 'regala pescado a un hombre y le darás alimento para un día, enséñale a pescar y lo alimentarás para el resto de 
su vida", por ende "el educador debe pensar en la imposibilidad de ser mero transmisor mediante la comunicabilidad que se establece al interior del proceso enseñanza-aprendizaje" (Prieto Castillo, 1999: 38), por ello cuando se habla de la pertinencia de los métodos de enseñanza, inmediatamente se hace una proyección hacia el desempeño que tendrá el estudiante, no solo por los conocimientos que aprende, sino por las reglas que adquiere para desenvolverse en su contexto.

Es normal que antes de usar un determinado método de acuerdo al estilo de aprendizaje y, logrando una correspondencia con la asignatura, se plantee en un primer instante los objetivos de la misma; esto va a guiar todo el proceso posterior de enseñanza, el uso de métodos, recursos, estrategias, técnicas, con la finalidad de que los discentes aprendan. De igual forma, es pertinente mencionar que "en el nuevo marco europeo entra en juego una importante variable previa a la definición de los objetivos: las competencias" (Ríos Hilario, 2009: 501), lo que se resume en una pequeña expresión: 'saber obrar en un contexto'.

Llevar a cabo un proceso metodológico va a depender mucho de la edad de los estudiantes, los objetivos propuestos, la asignatura a impartir, el contexto y sobre todo, "la identificación de los estilos de aprendizaje" (Salas Silva, 2008: 21), como resultado el docente deberá tomar en cuenta estas condiciones para llevar a cabo su labor de enseñanza. Es muy común pensar que "al terminar el proceso educativo de relación pedagógica, éste educando haya interiorizado actitudes, capacidades y saberes, aunque ahora se les denomine destrezas" (Mendez, 2010: 6), pero por el hecho de ser destrezas, pasa a tener dominio de reglas, símil a los métodos, seguidamente entiende mejor su entorno y puede pasar del lenguaje típico (realidad) al lenguaje formal (pensamiento, ideas, conceptos).

En esta relación de enseñanza-aprendizaje también se encuentra el discente, estudiante, la comunidad educativa, etc. ${ }^{5}$, que a lo largo de la historia ha sido el centro de la educación, puesto que es el educando quien va a alcanzar, porque descansa sobre sí el futuro de la humanidad, los saberes de sus antepasados y los que están por acontecer. Esto solo puede significar una cosa, que el estudiante debe prepararse para "enfrentar las incertidumbres" (Morin, 1999: 39). El educando hace suyo lo que le enseñan y también acoge las formas en las que el docente lo educa, exactamente habría que señalar que hace suyos los métodos que más le agradan y por los cuales ha podido aprender eficazmente.

Es oportuno plantearse la idea de un docente dictando una clase magistral en la que hace uso de un método rígido, para el caso puede surgir la idea de una clase totalmente aburrida por parte del profesor, sin embargo, se torna en algo distinto si este método está centrado en el estudiantado con el uso de técnicas adecuadas y aprovechamiento de recursos didácticos. 
Es posible que unos pocos se aburran, pero no se aplica a todo el salón, ya que algunos estudiantes son más intuitivos, participativos, abiertos, dialogantes, deductivos, entre otros, por consiguiente se comprueba que no todos aprenden de la misma manera y tampoco valdría utilizar un mismo método para poder enseñar y aprender. Gardner "argumenta que inteligencia no es alguna realidad estática determinada desde el nacimiento y muy buen medida con exámenes estandarizados" (Díaz, 2006: 31), por lo que no se podría enseñar lo mismo, de la misma forma, a todos.

"No hay docencia sin discencia" (Freire, 2007: 23), esto significa que quien se dedica a la enseñanza también aprende al enseñar y en efecto, sucede lo mismo con quien aprende porque enseña al aprender; a raíz de este pensamiento surgen tres componentes: enseñar exige rigurosidad metódica, enseñar exige criticidad y enseñar exige reflexión sobre la práctica (Moreira, 2012: 58-59). La referencia práctica de la rigurosidad metódica se halla en la aproximación del educando a los objetos cognoscibles por lo que el docente es responsable de las herramientas que le son útiles a los estudiantes para adquirir nuevos conocimientos; de igual manera la criticidad es importante porque permite al educando hacer frente a diversos problemas que son propios de su cotidianidad; por último, la reflexión sobre la práctica es vital porque permite dar cuenta del proceso de aprendizaje, es decir, entender las razones por las que aprende una cosa y no otra, entender lo que el docente le enseña. A continuación se resume esta idea:

Figura 1

Métodos en la enseñanza-aprendizaje

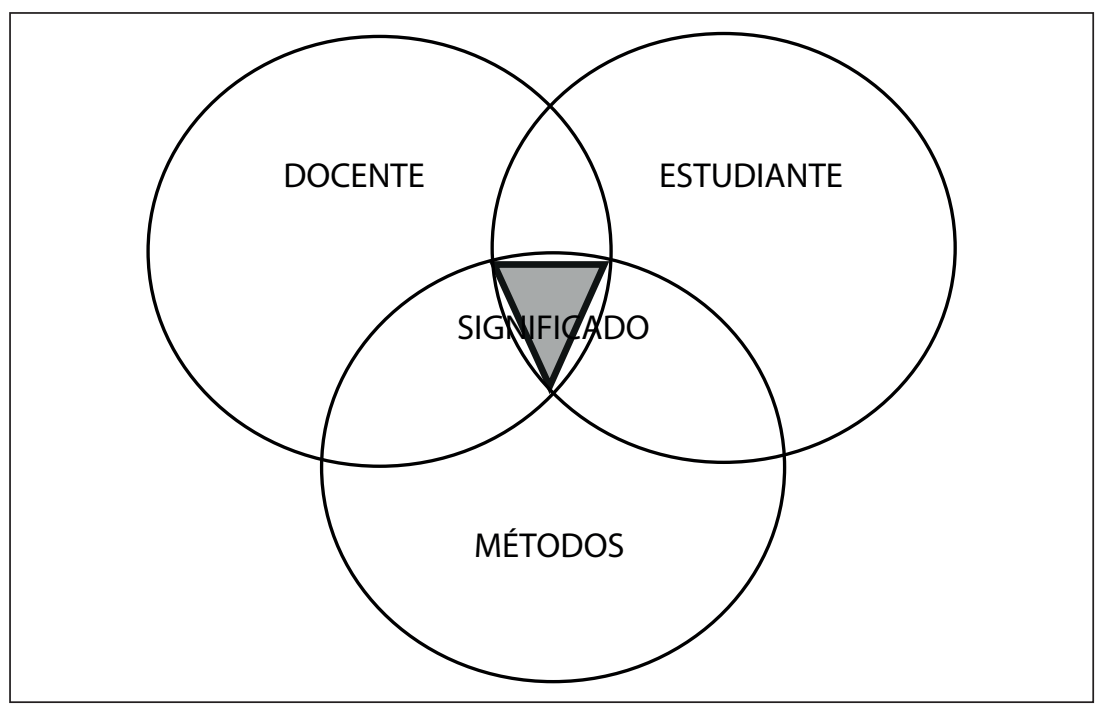

Elaboración: Jonathan A. Vivas Herrera 
La enseñanza se cumple a través del significado que el estudiante capta cuando el docente hace un apropiado uso de métodos para que haya un aprendizaje consciente y significativo. Se establece un punto de encuentro

¿Cómo es visto el proceso metodológico en esta relación de enseñanza-aprendizaje? Para resolver esta cuestión se sugiere una breve semejanza entre los métodos y las estrategias para entender mejor esta relación educador-educando, ya que a pesar de sus diferencias permiten alcanzar un mismo fin en la educación, por lo tanto "las estrategias didácticas que utilizan los docentes [...] son modificadas con el fin de dar respuesta a las dificultades de aprendizaje de los alumnos constituyen un aspecto más dinámico y cambiante de las prácticas docentes" (Álvarez, et al., 2008: 87), esto confirma que los métodos no pueden ser los mismos todo el tiempo, de modo que se debe dinamizar su utilización para mejorar la práctica docente, y a la vez para que haya un aprendizaje significativo, en términos de competencia.

Para que el estudiante aprenda, son necesarias condiciones estratégicas, antes de hacer uso de métodos de enseñanza porque a raíz de los estilos de aprendizaje se hace necesario hacer un diagnóstico con los estudiantes. Entre las condiciones estratégicas se presentan:

1) La identificación de las características socio-cognitivas del sujeto de aprendizaje.

2) El desarrollo de procesos de aprendizaje de complejidad progresiva.

3) La necesidad de determinar los conocimientos a aprender según características de los diferentes grupos de poblaciones y los tiempos disponibles.

4) El reconocimiento de las posibilidades de elección en el proceso de enseñanza.

5) Las relaciones sociales basadas en la cooperación, el desarrollo de la autonomía individual y el reconocimiento de la diversidad; y

6) La resolución de problemas como un esquema general que provee una secuencia de trabajo posible otorga sentido y direccionalidad a los procesos de enseñanza y de aprendizaje (Álvarez, et al., 2008: 92-94).

La comunicación que existe entre el docente y el educando es fundamental, considerando los pre-requisitos expuestos, surge una facilidad para la aplicación de métodos tanto de enseñanza como de aprendizaje, alcanzando así, condiciones óptimas para mejorar la calidad de la educación y un aprendizaje significativo. En este punto, se insiste en la calidad porque el estudiante acoge herramientas o reglas metodológicas para alcanzar un mayor grado de competencia educativa, de modo que puede aprender nuevas cosas por las habilidades y destrezas adquiridas como se muestra en las posteriores reflexiones. 


\section{Cancelación de la competencia educativa por la limitación del método}

Es erróneo pensar en un camino determinado, por el uso de un método específico, para alcanzar el conocimiento. No se puede limitar el conocimiento por la utilización de un método, ya que los individuos tienen diferentes estilos de aprendizajes y el método podría adaptarse de acuerdo a los estilos. Parecería poco oportuno explicar todos los métodos debidos a la gran cantidad que existen, así como los conocimientos que un individuo desea aprender. Para aprender literatura hay métodos que son distintos a los que se utilizan para aprender matemáticas, así mismo quien posee un estilo de aprendizaje visual no aprende de igual manera como quien aprende kinestésicamente, por esa razón se considera una limitación exponer un número finito de métodos.

Por la misma necesidad que se plantea lo anterior también se defiende la competencia educativa, ya que no se está tomando la competencia como en una competición sino como una habilidad y destreza que se enmarcan en la aptitud para ser, hacer y conocer. Es por eso que las reflexiones en relación a los métodos de enseñanza-aprendizaje son propicios para ampliar la visión que se tiene en correlación a la educación.

Reflexionar sobre la competencia educativa en el siglo XXI, exige tomar este término cuidadosamente, puesto que se suele confundir con su utilidad en el ámbito empresarial; por ello, hay que considerar competencia desde el punto de vista de la actividad, por lo que Piaget la considera importante para generar "esquemas mentales (el conocimiento no viene de los sentidos, sino de la acción sobre el mundo)" (Piaget, 1983: 8), esto se entiende a partir del desenvolvimiento que un individuo pueda tener frente a determinadas circunstancias. Podría ser que una persona adulta tiene un cúmulo de conocimientos, pero no sabe cómo responder en su condición humana porque no es capaz de dar solución a determinados problemas, ya sea las crisis a nivel económico de su país $\mathrm{u}$ hacer propuestas innovadoras de emprendimiento; al no dar respuesta ocasionaría un declive económico para toda la nación o no podría ser apto como emprendedor; desde esa suposición podría decirse que no hay competencia, pues tendría que saber intervenir para dar solución a este tipo de circunstancias. Se entiende que:

El término competencia se ha extendido desde sus orígenes, a varias disciplinas humanas con un sentido amplio de "conocimiento", "saber", "capacidad", referido en ocasiones no sólo al estrictamente lingüístico verbal sino a los correspondientes a los muchos otros códigos de comunicación (Grimaldi Herrera, 2009: 8). 
La competencia es un término que fue utilizado en primera instancia por los lingüistas ${ }^{6}$ para dar razón del dominio de una lengua por parte del hablante. Está muy relacionado con el performance o actuación, de manera que no se trata de dominio solamente; esa noción queda superada en el ámbito educativo para dar lugar a la incorporación de conocimiento, saber y capacidad, en una sola palabra podría llamarse 'destrezas', las cuales hacen del individuo un ser competente, pero no por competencia con un tercero, sino más bien por la capacidad para 'enfrentar las incertidumbres' (Morin, 1999), saber desenvolverse en su entorno, comprender el mundo, transformarlo y entenderse a sí mismo, inmerso en él. Tener dominio sobre reglas para actuar.

En todo este trabajo se venía haciendo uso de las palabra 'individuo' y 'sujeto', utilizados a propósito para concluir que ambos términos no hacen posible la 'competencia' porque desembocan en el ente indiviso en sí mismo y diviso de otro ser. Esta "indivisibilidad del individuo se afirma como incomunicabilidad e irrepetibilidad de su esencia individual $y$, por tanto, es totalmente en sí mismo y no en la parte de otro" (Saavedra, 2007: 140-141). Lo correcto se instaura en la persona y se vuelve competente porque es recíproca, se comunica, es empática, dialogable y sobre todo, la competencia es un hecho porque la persona "tiene la potencialidad de relación con el otro" (2007: 141).

No se puede cancelar la competencia educativa por la limitación del método porque el docente y el estudiante no son dos realidades aisladas, funcionan como un todo; por un lado está el docente que debe tener las competencias necesarias para enseñar y por el otro, el estudiante, quien es el que debe volverse competente para seguir aprendiendo y enfrentar las incertidumbres. De igual manera sucede con los métodos de enseñanza, en la aplicación se observa su utilidad porque el docente adquiere destrezas, habilidades, se vuelve competente para enseñar y procura que el estudiante aprenda, no solo conocimientos, a modo de recipiente, sino para la vida. Por tal motivo se afirma que:

Los docentes -guías, tutores, acompañantes y mediadores de aprendizajes- son quienes tienen la responsabilidad de influir en el desarrollo del pensamiento de los niños, haciéndoles emitir sus propias ideas, aunque estas no concuerden con el tema propuesto, pues la operación mental se da y la práctica de ésta hará que la persona, desde la infancia, se desenvuelva con fluidez en cualquier circunstancia de la vida (Jara, 2012: 61).

El docente también tuvo que atravesar un proceso semejante al que ahora ejecuta, por tanto conoce cómo funciona un ambiente educativo. A veces se corre el riesgo de conformarse con lo que se conoce, por eso surge la necesidad de actualización constante para hacer frente a los retos que se 
imponen en la enseñanza. La historia demuestra que cada época ha visto nacer a nuevas generaciones, con distintos requerimientos, buscando otros sueños; la sociedad progresa y hay cambios significativos por lo que el docente, en términos de competencia, debe estar en continuo proceso de aprendizaje para enseñar pertinentemente a sus estudiantes.

La competencia educativa se inserta en la relación docente-discente a partir de las implicaciones que se dan para la cognición. Se hace mención a las reflexiones de Sanmartín sobre el 'El contenido de la cognición incorporada', donde "la tarea de la cognición incorporada es evitar pensar ignorando los escalones previos del conocer, similar a un proceso de pensar sin saber cómo se conoce" (2011: 135), efectivamente el educando habrá de comprender el cómo ha adquirido los conocimientos que se le enseñan, así como el camino que ha seguido para aprender. Luego, este "sujeto no es un núcleo que conduce y procesa información, sino que se convierte en el punto donde se metaboliza el conocer" (Sanmartín, 2011: 135), comprende lo que conoce y también lo asimila; entiéndase esto último semejante al metabolismo, donde algo que ingresa se desintegra y fortalece diversas áreas del cuerpo, convirtiéndose en proteínas.

Si los docentes se vuelven competentes, también lo harán los estudiantes porque la plasticidad de su cerebro lo hace posible, de modo que adquieren conocimientos como también las reglas para seguir conociendo:

Podemos decir que la construcción del conocimiento escolar es en realidad un proceso de elaboración, en el sentido de que el alumno selecciona, organiza y transforma la información que recibe de muy diversas fuentes, estableciendo relaciones entre dicha información y sus ideas o conocimientos previos (Díaz Barriga Arceo \& Muriá Vila, 1998: 35).

En definitiva, el estudiante, aparte de entender cómo aprende, también se entiende a sí mismo en relación con su entorno. La competencia se evidencia cuando sabe relacionarse con el otro, con su entorno, de modo que puede resguardar los bienes presentes para el futuro de la humanidad (Morin, 2011). Hacer un uso consciente de lo que se posee, para ayudar a otros y darle sentido a la propia existencia en ese devenir. El hombre busca humanizarse, siendo este el fin último de la educación.

Los métodos de enseñanza-aprendizaje pueden considerarse como parte del esqueleto transversal de la educación por lo que hace posible la consecución de los fines de la misma. La humanización del hombre se instaura en un proceso que debe llevarse a cabo con cuidado, es decir, pertinentemente para cumplir con los objetivos propuestos. En Ecuador se incluye en el Buen Vivir, por lo tanto todos los esfuerzos educativos estarán orientados hacia ese fin. 


\section{La teleología de la educación, resultado de un proceso pertinente}

La teleología de la educación hace posible entablar un diálogo sobre los distintos modelos pedagógicos, de igual manera permite emitir un juicio de valor sobre los métodos de enseñanza-aprendizaje como medio para el cumplimiento de la finalidad; para comprobar esta defensa se expone lo siguiente:

Toda praxis educadora -familiar, escolar u otra- está inserta en una antropología filosófica desde la cual se vuelve coherente e inteligible, lo cual, sin embargo, no quiere decir que quede justificada. En esto no hay justificación científica posible quedando siempre el último reducto en manos de la libertad de cada quien, la cual decidirá -si hay decisión- en última instancia (Fullat, 2002: 231).

La praxis educativa comprueba que, cuando no hay libertad, tampoco es posible que haya una elección antropológica, en consecuencia se cae en un reduccionismo de la práctica educativa a un conjunto de mecanismos neuro-psicosociológicos (Fullat, 2002). Se observa que con libertad o sin ella, la educación, antes que nada, tiene una finalidad y se inserta en la humanidad del hombre. Las limitaciones impiden alcanzar los fines propuestos a nivel educacional, así como también dejar las decisiones en las manos de cada quien. Esto no puede ser educación.

El camino correcto se establece a partir de la introducción de valores en el terreno educativo, es por eso que no hay métodos buenos ni malos sino que depende del cómo sean puestos en práctica, se hace un uso correcto del juicio de valor en base a la relación enseñanza-aprendizaje y una de muchas soluciones posibles, por lo que se ha revisado, es que la pertinencia se ajusta a la vía que conduce al 'telos'.

A partir de este instante habrá buenas y malas educaciones, según sea la axiología desde la cual uno se pronuncia. No hay verificación de la bondad o maldad axiológicas de un proceso educador. [...] No es posible situarse en el punto de vista de los valores totales, como si uno fuera Dios. Quien tal hace es un dogmático [...], es un dogmático que encubre así su talante totalitario (Fullat, 2002: 232).

El único ser que puede educarse es el hombre y hacia él deben estar avocados todos los esfuerzos de educación, de modo que sea más humano en la medida que es consciente de su existencia, le da un sentido a su relación con otros y se da cuenta de que es parte de un todo para poder cambiar su realidad, por consiguiente:

La educabilidad es una posibilidad y una categoría humana, fundamento de la acción educadora. Significa viabilidad del proceso educativo y 
la afirmación de que la educación es factible en la persona, básicamente por la condición de su plasticidad y ductibilidad, que le permite recibir influencias y estímulos y reaccionar ante ellos, con lo cual elabora nuevas estructuras que lo personalizan y socializan, o, la capacidad que la persona tiene de adquirir nuevas conductas, de perfeccionarse y transformarse (Di Caudo, 2007: 96).

El valor de ser 'persona' no puede concebirse como una cuestión material la cual puede manipularse como en el caso de la ciencia, por esa razón el estudiante toma consciencia de lo que aprende, hace uso de reglas que le permiten un andamiaje más fuerte. Se pasa del plano físico a lo metafísico y se acepta que el valor humano es trascendente y que por consiguiente no puede someterse a nada; tal alcance del valor humano, mueve a entender con mayor precisión el carácter trascendente de los valores. Sin trascendencia, los valores se achatan, se desvirtúan y pierde su verdadera densidad humana. Partiendo de la concepción del hombre en su totalidad y unidad, a partir de una replantación de la reflexión del hombre y a la vez, por medio de lo que intelige podría situarse en el mundo logrando de este modo que en una era actual como lo es la postmodernidad se pueda hablar de una ética de la persona, más no de algo pasajero; lo cual es el espacio en donde se ha instaurado todo lo que ha originado la ciencia y que a su vez provee una felicidad temporal.

La teleología no solo tiene que ver con el uso de métodos, pues ésta engloba a toda la educación aunque, en defensa de lo que es pertinente o no, es viable aceptar que los docentes son responsables del futuro porque son quienes educan a los niños que se convertirán en hombres y mujeres del mañana; quienes deben velar por el bienestar de toda la humanidad. La persona que aprende se forma en relación a determinados valores que se aúnan al conjunto de reglas que domina, sintetizados en habilidades y destrezas para la toma consciente de decisiones y para transformar su entorno.

Los valores no se oponen a la realidad sino que son un aspecto de ese trozo de realidad elaborada por todos y se conoce como experiencia; a lo sumo se puede decir que los valores no son cualidades actuales sino potenciales, esto es, disposiciones, pero a este respecto no difieren de disposiciones físicas tales como 'pesado' o 'frágil' (Bunge, 1997: 16). En pocas palabras se comprueba que el conocimiento exige un perfil al hombre, de manera que se prefigura para algo, para cambios, para la vida, para existir; desde ahí se piensa en la pertinencia de lo que se enseña y lo que se aprende. El currículo nacional, a nivel macro y micro habrá de responder a este postulado; los contenidos, métodos, recursos, técnicas, estrategias, objetivos, entre otros, instan a la humanización del hombre.

Por último, el lugar en el que converge enseñanza-aprendizaje, subyace no sólo en el aula, sino que se parte de la educación ya sea en la 
familia, la sociedad, el entorno y, es el docente quien ayuda a establecer el criterio para determinar lo que está bien o mal, de acuerdo a valoraciones universales y consensuadas. Luego, habrán quienes decidan regirse por intereses personales, sin embargo siempre hay máximas ${ }^{7}$ para decidir lo que humaniza o lo que deshumaniza al hombre. Seguir un proceso metodológico de enseñanza-aprendizaje es considerado una herramienta valiosísima para conocer la realidad, entender el mundo y comprenderse a sí mismo en él.

\section{A modo de conclusión}

Las nuevas disciplinas científicas están desentrañando secretos acerca del cerebro que antes se desconocían y no se puede culpar a nadie por padecer ese estado de ignorancia, más bien la ciencia ha seguido un proceso propicio de maduración para dar a luz nuevos descubrimientos y facilitarnos el entendimiento de lo que antes no se comprendía. En resumen la ciencia busca acercarse a una verdad de carácter objetivo, pero es necesario humanizar la ciencia para beneficio de la sociedad. Consecuentemente se puede observar que el hombre busca hacerse de nuevos conocimientos y por ello, sigue determinados métodos para lograr su cometido, el estudio de la mente comprueba ello, además de que no todos los seres humanos aprenden de la misma forma (Díaz, 2006: 33).

Los métodos de enseñanza-aprendizaje no son algo sobre lo que se debe reflexionar una sola vez y listo, eso sería un error, pues como se dijo anteriormente, la sociedad avanza y se encuentra en continuo cambio, por lo cual los docentes deben diversificar sus métodos sin perder de vista el fin último de la educación. Análogo a esto, los estudiantes también aprenden y procuran adoptar los conocimientos aprendidos, así como las reglas que adquieren para apropiarse de nuevos saberes, algo semejante como obtener destrezas y habilidades para volverse competentes y transformar su entorno (Freire, 2007: 62), siempre y cuando lo requiera.

Por eso, cualquier método de enseñanza debe representar un sistema de acciones del maestro dirigidas hacia un objetivo que organice la actividad cognoscitiva y práctica de los alumnos, la cual garantiza que los mismos asimilen el contenido de la enseñanza.[...] Ningún método puede considerarse universal y apropiado para resolver todos los problemas docentes. La condición de la efectividad del proceso de enseñanza-aprendizaje de cualquier asignatura y en particular de la física, es la aplicación de diferentes métodos en dependencia de los objetivos de la clase, de las características de los alumnos y otros. Ningún método se aplica puro y aislado de los demás (Herrera Fuentes, 2012: 6). 
Es a raíz de la asimilación desde donde inicia la competencia educativa, puesto que las acciones de los docentes permiten afianzar los conocimientos y hacen posibles los aprendizajes futuros. Es una prioridad no limitar los métodos porque hay que experimentar, pero objetivamente, ya que de no ser así, por el hecho de tomar decisiones libres, se puede caer en reduccionismos que imposibilitan mejorar la calidad de la educación y obstaculizan los aprendizajes.

En última instancia se prevé que todos los esfuerzos educativos estén orientados a la humanización del hombre y eso es el resultado de la pertinencia educativa, puesto que no se puede dejar a la libre decisión que se enseñe lo que sea, que se haga uso de cualquier medio y mucho menos que se siga considerando al hombre como individuo. Esto coarta un verdadero aprendizaje que dé sentido a la existencia de cada persona. Desde la educación se debería posibilitar una estructuración cognitiva fuerte que conlleve la relación con los otros y la búsqueda del bien común.

\section{Notas}

1 Se refiere a la desambiguación, es decir, a una tablilla en la que no se encuentra escrito absolutamente nada y está dispuesta para inscribir, por un tercero, lo que se desee en ella. En la educación esta idea se encuentra muy vinculado con el conductismo.

2 En síntesis se recoge una clara idea de las experiencias que un individuo tiene en su proceso de escolarización; al compartir con sus compañeros de clase se da cuenta de la riqueza que hay en la diversidad de pensamiento porque todos aprenden y estructuran sus ideas de acuerdo a sus motivaciones y exigencias del contexto.

3 Este refrán perteneciente a la cultura china, ha sido utilizado durante siglos para el mejoramiento de la educación y al mismo tiempo ha sido un baluarte para la elaboración de libros con fines educativos. Su pedagogía hace pensar en el interés que debe tener el educador para con sus educandos, en tanto que ellos son los actores principales en la educación.

4 La palabra estilo fue utilizada por Gordon Allport en una de sus obras, por los años de 1930, para definir las pautas consistentes que aparecían en los individuos. Siete años después, según Keefe (1979), Allport acuñó el término de 'estilo cognitivo' como una referencia a la 'calidad de vida y de adaptación manifestada por los distintos tipos de personalidad' (Salas Silva, 2008).

5 Se exponen las diferentes formas de llamar al estudiante porque cada palabra ha adoptado una forma distinta de ver al educando a lo largo de la historia de la humanidad, ya que cada paradigma educativo ha comportado una forma distinta de significar a quien aprende.

6 El término competencia fue utilizado por los lingüistas de mediados del siglo $\mathrm{XX}$, aunque fue Chomsky quien le dio un sentido más amplio en sus trabajos de Gramática Generativa.

7 Refiérase a máximas universales o imperativos categóricos que han sido superados, sin embargo, hay quienes equívocamente hacen uso de ellos para superponerlos a los valores inherentes al ser humano. 


\section{Bibliografía}

ÁlVAREZ, Mónica, ALZAMORA, Sonia, DELGADO, Verónica, GARAYO, Perla, MORENO, Verónica, MORETTA, Rosana, \& NEGROTTO, Adolfo

2008 Prácticas docentes y estrategias de enseñanza y de aprendizaje. Educación, Lenguaje y Sociedad, V(5), 81-106. Diciembre.

AUSUBEL, David P.

2002 Adquisición y retención del conocimiento: Una perspectiva cognitiva. Barcelona: Paidós Ibérica, S.A.

BRUNER, Jerome

1963 El proceso de la educación. México D.F.: UTEHA.

BUNGE, Mario

1997 Ética, ciencia y técnica. Buenos Aires: Editorial Sudamericana.

DI CAUDO, María Verónica

2007 La Construcción de los Sujetos de la Educación. Sophia: Colección de Filosofía de la Educación, II, 91-132. Junio.

DÍAZ BARRIGA ARCEO, Frida, \& MURIÁ VILA, Irene

1998 El desarrollo de habilidades cognoscitivas para promover el estudio independiente. Tecnología y Comunicación Educativas, 29-43.

DÍAZ LEFEBVRE, René

2006 Inteligencias múltiples. Obregón: Orbis Press.

FREIRE, Paulo

2007 Pedagogía da Autonomía: saberes necessários à prática educativa. São Paulo: Paz e Terra.

FULLAT, Octavi

2002 Entrevista a Octavi Fullat I Genís. (S. Rudzki, entrevistador), 23 de junio.

GRIMALDI HERRERA, Carmen

2009 Competencia lingüistica y competencia comunicativa. Recuperado el 20 de Julio de 2012, de Contribuciones a las ciencias sociales: http://www.eumed. net $/ \mathrm{rev} / \mathrm{cccss} / 06 / \mathrm{cgh} 2 . \mathrm{htm}$

GUILAR, Moisés Esteban

2009 La ideas de Bruner: 'de la revolución cognitiva' a la 'revolución cultural'. Educere, 13(44), 235-241. Marzo.

HERRERA FUENTES, Julián

2012 Casanchi. Obtenido de Métodos de Enseñanza-aprendizaje, febrero.

JARA, Victoria

2012 Desarrollo del pensamiento y teorías cognitivas para enseñar a pensar y producir conocimientos. Sophia: Colección de Filosofía de la Educación, XII, 53-66.

MÉNDEZ, Zayra

2010 Aprendizaje y cognición. Costa Rica: UNED.

MOREIRA, Marco

2012 Aprendizaje significativo, campos conceptuales y pedagogía de la autonomía: Implicaciones para la enseñanza. Meaningful Learning Review, II(1), 44-65.

MORIN, Edgar

1999 Los siete saberes para la educación del futuro. París: UNESCO.

2011 La vía para el futuro de la humanidad. Barcelona: Espasa Libros, S.L.U.

PIAGET, Jean

1980 Adaptación vital y psicología de la inteligencia (Tercera ed.). México: Siglo Veintiuno Editores, S. A. 
1983 Esquemas de acción y aprendizaje del lenguaje. En: Centre Royomount Pour une Science de l'homme. Teorías del lenguaje, teorías del aprendizaje: el debate entre Jean Piaget y Noam Chomsky. Barcelona: Crítica.

PRIETO CASTILLO, Daniel

1985 Discurso autoritario y comunicación alternativa. México: EDICOL.

1999 La comunicación en la educación. Buenos Aires: Ediciones CICCUS-La Crujía. RÍOS HILARIO, Ana B.

2009 De los métodos didácticos tradicionales a los métodos europeos: experiencias de la asignatura piloto de Catalogación Descriptiva. En: A. García-Valcárcel Muñóz-Repiso, Experiencias de Innovación Docente Universitaria (pp. 499-506). Salamanca: Ediciones Universidad Salamanca.

ROLÓN, Adela, PAEZ, Julio, SAINT-ANDRÉ, Estela, MARTÍN, Alicia V., \& LEAL, Eugenia

1997 Apropiación del conocimiento: Interdiscursividad: Filosofía del Lenguaje, Filosofía de la Literatura y Educación. San Juan - Argentina: effha.

SAAVEDRA, Alejandro

2007 El cuarto hombre. Sophia, II, 133-164. Junio.

SALAS SILVA, Raúl Ernesto

2008 Estilos de aprendizaje a la luz de la Neurociencia. Bogotá: Cooperativa Editorial Magisterio.

SANMARTÍN, Rómulo

2011 La cognición incorporada: El contenido y la justificación del enfoque percepto-operacional del conocimiento. Sophia: Colección de Filosofía de la Educación, $X, 127-166$, julio.

2014 Estructuración filo y ontogenética de la cognición incorporada. Sophia: Colección de filosofía de la educación, XVI, 121-168, enero-junio.

Fecha de recepción del documento: 5 de julio de 2015

Fecha de aprobación del documento: 18 de septiembre de 2015 